\title{
PRINCIPAL IDEALS IN THE IDEAL LATTICE
}

\author{
H. SUBRAMANIAN
}

ABSTRaCt. We show that there cannot be a definition of "principal elements" in the theory of multiplicative lattices so that the notion of principal elements concurs with the notion of principal ideals when interpreted in the ideal lattices of rings.

A lattice $L$ together with a binary associative multiplication satisfying $x y \leqq x \wedge y, x(y \vee z)=x y \vee x z$ and $(y \vee z) x=y x \vee z x$ is called an $m$-lattice. For an arbitrary (associative) ring $R$, the lattice $L(R)$ of (two-sided) ideals of $R$ is the motivating example of an $m$-lattice, the multiplication being the ideal product. $L(R)$ is also residuated, the residual associated with the ideal product.

R. P. Dilworth [1] has defined principal elements in residuated $m$-lattices, and has shown that this characterizes the principal ideals of a U.F.D. He also remarks that "in more general rings there may be many nonprincipal ideals which are principal elements of the lattice of ideals". The cited example is the ring of integers in an algebraic number field. This raises the question whether it is possible at all to spot the principal ideals of a ring $R$ in the $m$-lattice $L(R)$. Taking the cue from Dilworth's remark, we show that this is impossible. We proved a lesser statement in [2].

Let us consider the Dedekind domain $D=\left\{m+n(-5)^{1 / 2} \mid m, n \in Z\right\}$. For any $m, n \in Z$ the ideals $(m)$ and $(n)$ of $D$ are equal if and only if $m= \pm n$. Thus $D$ has infinitely many ideals. But $D$ being countable and Noetherian, there are only countably many ideals of $D$. In any Dedekind domain, every nonzero proper ideal is uniquely a finite product of maximal ideals. So $D$ has countable infinitely many maximal ideals. Now take any bijection $\varphi$ between the sets of maximal ideals of $D$ and $Z$, and extend it uniquely to an isomorphism $\bar{\varphi}$ between the semigroups (by ideal product) $L(D)$ and $L(Z)$. Since the ideal sum and ideal intersection in any Dedekind domain are induced by the ideal product, $\bar{\varphi}$ is indeed an isomorphism between the $m$ lattices $L(D)$ and $L(Z) . D$ is not a P.I.D., but $Z$ is; and hence our conclusion.

\section{REFERENCES}

1. R. P. Dilworth, Abstract commutative ideal theory, Pacific J. Math. 12 (1962), 481498. MR 26 \#1333.

2. H. Subramanian, On theory of $x$-ideals, J. Algebra 12 (1969), 134-142. MR 39 \#1385.

State University of New York at Buffalo, Amherst, New York 14226

Received by the editors January $11,1971$.

AMS 1970 subject classifications. Primary 06A20, 13 F05.

Key words and phrases. Dedekind ring, ideal lattice, principal ideal, $m$-lattice.

(c) American Mathematical Society 1972 